\title{
Thermal Comfort Analysis of an Automobile Driver with Heated and Ventilated Seat
}

\author{
G. Karimi, E.C. Chan, and J.R. Culham \\ Department of Mechanical Engineering, University of Waterloo \\ Waterloo, ON, Canada, N2L 3G1 \\ I. Linjacki and L. Brennan \\ W.E.T. Automotive Systems Ltd., Windsor, ON, Canada, N8N 5B8
}

Copyright $\odot 2002$ Society of Automotive Engineers, Inc.

\begin{abstract}
A thermal/physical model of the dynamic interaction between an automobile passenger, the cabin environment, and a heated/ventilated seat is presented. The model considers the human body as being made of 21 distinct segments and three-layers. Simple mathematical models are presented to simulate heating and ventilation of cool air through the seat.
\end{abstract}

The model has the ability to predict the transient response of a driver in a highly non-uniform thermal environment in terms of local and overall thermal comfort levels.

\section{INTRODUCTION}

The cabin temperature of an automobile can exceed $80{ }^{\circ} \mathrm{C}$ on a hot summer day due to incident solar radiation, while during harsh winter conditions, ambient temperatures can drop below $-30^{\circ} \mathrm{C}$. Under this extreme range of ambient conditions, automobile passengers can experience shocking localized heating or cooling as exposed body surfaces (15 to $20 \%$ ) make contact with the seat, back support and steering wheel. Although the heating and air-conditioning systems within an automobile attempt to respond to the comfort needs of passengers, the thermal capacity of most cabin components limits the timely response of these heating and ventilation systems, resulting in passenger discomfort for extended periods. While the ambient air temperature is an important factor in determining the level of thermal comfort, conductive heat transfer from the body due to contact with a seat that is initially very cold or very hot plays a significant role in influencing the thermal sensation of an automobile passenger.

The ability to reach thermal neutrality over the contact area during the cold season, can be expedited by incorporating resistive heating elements into the seat to augment the standard engine-coolant-based heating system. A possible strategy to improve the cooling process is to ventilate the seat with ambient air from the passenger compartment. This technique can substantially reduce the time lag between heat transfer from the ambient air to the seat contact area. Furthermore, it can enhance the comfort level in the microclimate in the contact area.

Although automobile car seats with heating elements have been in the market for several years, ventilated seats are a more recent development, which require further attention.

In this paper, physical models are presented to simulate the thermal interactions between a typical driver, and a heated or ventilated seat. The effectiveness of the seat for enhancing thermal comfort under severe winter and summer conditions will be examined.

\section{DRIVER'S THERMAL RESPONSE MODEL}

There are two stages in thermal comfort modeling. The first stage accounts for the heat exchange process between the body and the surrounding environment in order to estimate the rate of heat storage and body temperature. The second stage uses the information obtained in the first stage to predict the thermal sensation (comfort) the person would experience.

Several models that consider the thermal interaction of the human body with the environment have been developed during the past 50 years. Excellent reviews and critical evaluations of these models can be found in Dohetry and Arens (1988), Haslam and Parsons (1988), and Lotens (1988). 
A more detailed thermal analysis can be obtained by considering the body to be made of different layers and multiple segments.

The human thermal response model developed in this work is based on the model of Burch et al. (1992). The model considers the body to be made of three layers: core, skin and the clothing worn. The major difference in the present study is in the number of human body segments, where the human body is divided into 21 distinct segments, as shown in Fig. 1 and described in Table-1.

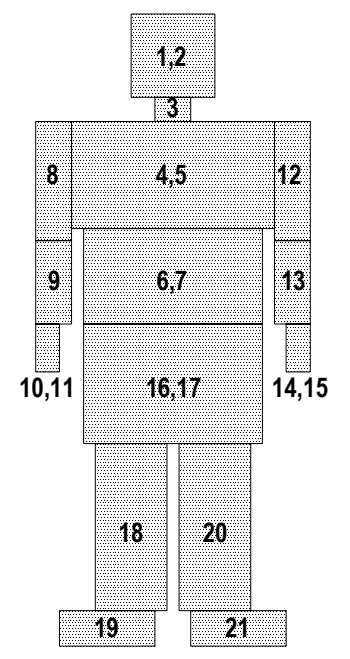

Fig. 1: The human body segments

The selection of body segments, as shown in Fig. 1, provides local as well as overall thermal comfort estimation of the human body for a wide range of clothing and environmental conditions. For example, the subject can wear short sleeve shirts and shorts as well as regular clothing. In addition, the effect of heating or ventilation through the driver seat, the steering wheel, and local air velocity can be investigated.

The thermal resistance network of a typical clothed human body segment, as approximated by this model is illustrated in Fig. 2. As shown, heat (per unit area) is generated in the body by normal metabolic performance, $\mathrm{q}_{\mathrm{MT}}$, and can be lost to the environment by conduction, $\mathrm{q}_{\mathrm{CN}}$, convection, $\mathrm{q}_{\mathrm{CV}}$, radiation, $\mathrm{q}_{\mathrm{RD}}$, respiration, $\mathrm{q}_{\mathrm{RS}}$, and evaporation of sweat from the skin, qEP. The rate of heat storage in the body segment is the difference between the rate of heat generation and the rate of heat loss to the environment.
Table-1: Body segments

\begin{tabular}{ll}
\hline No. & Description \\
\hline 1 & Head face \\
2 & Head back \\
3 & Neck \\
4 & Chest \\
5 & Upper back \\
6 & Stomach \\
7 & Lower back \\
8 & Right upper arm \\
9 & Right lower arm \\
10 & Right hand (back) \\
11 & Right hand (palm) \\
12 & Left upper arm \\
13 & Left lower arm \\
14 & Left hand (back) \\
15 & Left hand (palm) \\
16 & Upper leg (front) \\
17 & Upper leg (back) \\
18 & Right lower leg \\
19 & Right foot \\
20 & Left lower leg \\
21 & Left foot \\
\hline
\end{tabular}

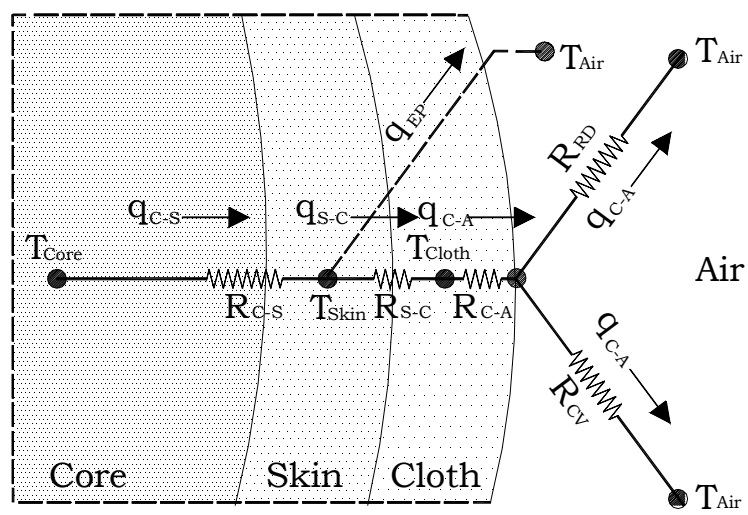

Fig. 2: A typical clothed body segments with thermal resistances

Fundamental heat transfer principles can be used to evaluate the dry heat loss (conduction, convection and radiation) in terms of skin temperature, $T_{S K}$, the thermal resistances, $R_{i} S$, and environmental conditions $\left(T_{A}, T_{R D}\right.$, $V_{A}$, and $\left.P_{A}\right)$. Skin temperature, $T_{S K}$, and heat fluxes $q_{E P}$, and $\mathrm{q}_{\mathrm{RS}}$ are dependent on complex thermo-regulatory functions of the body including sweating, shivering, and the control of the blood flow through vascular constriction and dilation. In general, these thermo-regulatory functions vary over the body in response to changing rates of heat loss due to thermal transients and nonuniformity in clothing, temperature, and air velocity. 
The equations for each of the heat gain/loss terms are presented and heat balances are made on each node in order to develop equations for estimating the temperature of the nodes as a function of time.

\section{BODY INTERNAL HEAT GENERATION}

Under normal conditions, heat is generated in the body core only from the metabolism inherent to a given physical activity. The amount of energy released by the metabolism is dependent on the amount of muscular activity. According to Fanger (1972), the average metabolic rate for an automobile driver varies between 1 and 1.8 met $\left(1 \mathrm{met}=58.15 \mathrm{~W} / \mathrm{m}^{2}\right)$ depending on the traffic level.

\section{HUMAN BODY HEAT LOSS}

In this section the heat loss from body segments due to different mechanisms are presented.

\section{Heat Loss by Conduction}

Heat exchange between the core and the skin occurs by conduction and by advection of blood. The heat transfer by advection is dependent on the rate of blood flow between the core and skin layers. The combined thermal exchange between the core and skin can be written as:

$$
\begin{array}{r}
Q_{C S, i}=\left\{\left(h_{B L}+\rho_{B L} \cdot C_{P, B L} \cdot \dot{v}_{B L}\right) A\left(T_{C R}-T_{S K}\right)\right\}_{i} \\
i=1,2, \ldots, N
\end{array}
$$

where $h_{B L}$ is the effective conductance between the core and skin (=5.28 W/(m $\left.{ }^{2} . K\right)$, ASHRAE, 1993). The specific heat and density of the blood are taken as $4,145 \mathrm{~J} / \mathrm{kg} . \mathrm{K}$ and $1,010 \mathrm{~kg} / \mathrm{m}^{3}$, respectively, and $A$ is the skin surface area. Equation 1 is used to calculate core-to-skin heat transfer for all body segments, $\mathrm{N}$.

The skin blood flow rate per unit of skin area, $\dot{v}_{B L}$, which is regulated by the thermo-regulatory system will be discussed later in this section.

Transfer of dry heat between the skin and the outer surface of a clothed body is quite complicated, involving internal convection and radiation processes in the clothing pores, and the conduction through the cloth itself. Generally, a heated manikin is used to accurately measure the effective thermal conductivity of different clothing ensemble.

Heat loss from the skin to the clothing, $Q_{s c}$, can be written as

$$
Q_{S C, i}=\left\{k_{C L} \cdot f \cdot A \cdot \frac{\Delta T}{\Delta x_{C L}}\right\}_{i} \quad i=1,2, \ldots, N
$$

where $k_{C L}$ is the effective thermal conductivity of the clothing ensemble and $\Delta \mathrm{T} / \Delta \mathrm{x}_{\mathrm{CL}}$ is the temperature gradient across the clothing. $f$ is a factor $(\geq 1)$ to correct for the heat transfer area from the skin to the external surface of the clothing.
Heat Loss by Convection

The heat loss by convection from the directly exposed skin and the outer surfaces of the clothed body can be expressed by the following equation:

$$
Q_{C V, i}=\left\{h_{C V} \cdot f \cdot A \cdot \Delta T\right\}_{i} \quad i=1,2, \ldots, N
$$

Here, $\Delta T$ is the difference between the skin temperature (or the clothing temperature) and the ambient air. $f$ is the ratio of the effective surface areas of the clothed body and the nude body. The convective heat transfer coefficient, $h_{\mathrm{CV}}$, is given by (ISO 7730)

$$
\begin{gathered}
h_{C V, i}=12.1 \sqrt{V_{A, i}} \quad \text { if } \quad 2.38 \Delta T_{i}^{0.25}<12.1 \sqrt{V_{A, i}} \\
h_{C V, i}=2.38 \Delta T_{i}^{0.25} \quad \text { Otherwise } \\
\quad i=1,2, \ldots, N
\end{gathered}
$$

Heat transfer between the clothing and the seat in the contact areas is governed by conduction and is calculated using Eq. 2.

Heat Loss by Radiation

Heat loss by radiation is given by

$$
Q_{R D, i}=\left\{\sigma \cdot \varepsilon \cdot F_{G} \cdot f \cdot A \cdot\left(T^{4}-T_{R D}{ }^{4}\right)\right\}_{i} \quad i=1,2, \ldots, N
$$

where $\sigma$ is the Stefan-Boltzmann constant, $5.67 \times 10^{-8}$ $\mathrm{W} /\left(\mathrm{m}^{2} \cdot \mathrm{K}^{4}\right) . \varepsilon$ is the average emissivity of clothing or body surface (about 0.9 for most cotton clothing and about 1.0 for the skin) and $F_{G}$ is the view factor, or the fraction of the skin or clothed area that radiates to the environment. Body segments are considered as simple planar elements parallel to the plane of surrounding surfaces and view factors are approximated based on the area fraction exposed to the surrounding surfaces (e.g. view factors are estimated at 1.0 for the head face, and head back, and 0.1 for the upper leg back, respectively).

Heat Loss by Evaporation of the Sweat

The total latent heat loss from the skin due to evaporation, $Q_{E P}$, is given by

$Q_{E P, i}=\left\{\omega \cdot A_{E f f} \cdot \frac{P_{S K}-P_{A}}{R_{M, S A}}\right\}_{i} \quad i=1,2, \ldots, N$

where $\omega$ is the total skin wetness (percentage of the exposed area which is moist), and $\mathrm{R}_{\mathrm{M}, \mathrm{SA}}$ is the total vapor diffusion resistance due to the clothing and the surrounding air. $P_{S K}$ and $P_{A}$ are water vapor pressures at the skin and ambient temperatures, respectively.

\section{Respiration Heat Loss}

Heat and water vapor are transferred to inhaled air by convection and evaporation during respiration. Assuming the air is exhaled at saturated conditions, the total sensible and latent heat due to respiration is equal to 
$Q_{R S}=\dot{m}_{A}\left[C_{P, A}\left(T_{C R}-T_{A}\right)+h_{f g} \frac{\left(P_{C R}-P_{A}\right)}{P_{t}}\right]$

Here, $P_{C R}$ and $P_{t}$ are the water partial pressure at the core temperature and the ambient pressure, respectively. $h_{\mathrm{fg}}$ is the water latent heat of vaporization. It is assumed that the respiration heat loss affects only the first five body segments hence; $Q_{R S}$ is divided among these segments proportional to their surface area.

The mass flow rate of air inhaled is estimated as a linear function of total metabolism (Burch et al. 1992):

$\dot{m}_{A}=1.3 \times 10^{-6} Q_{M T}$

\section{Human Thermo-regulatory System}

The human body has a very effective temperature regulatory system, which ensures that the body's core temperature is kept at approximately $37^{\circ} \mathrm{C}$. When the body becomes too warm, two processes are initiated: first the blood vessels dilate, increasing the blood flow through the skin and subsequently one begins to sweat. If the body is getting too cold, the first reaction is for the blood vessels to constrict, reducing the blood flow through the skin. The second reaction is to increase the internal heat production by stimulating the muscles, which cause shivering.

The control signal equations used in the present work are taken from Dohetry and Arens (1988). The parameters used in the control signals are the core, skin, and mean body temperatures. The mean body temperature, $T_{B}$, is a mass weighted average of the skin and the core temperatures.

The increase in the metabolism due to shivering is estimated by

$$
Q_{S H, i}=\left\{34.9\left(33.7-T_{S K}\right)\left(36.8-T_{C R}\right)\right\}_{i} \quad i=1,2, \ldots, N
$$

The blood flow between the core and the skin per unit of skin area is expressed as

$$
\begin{array}{r}
\dot{v}_{B L, i}=\left\{\frac{\left(1.75 \times 10^{-6}\right)\left(1+31.7\left(T_{C R}-36.8\right)\right)}{1+0.1\left(33.7-T_{S K}\right)}\right\}_{i} \\
i=1,2, \ldots, N
\end{array}
$$

The rate of sweat production per unit skin area, $\dot{m}_{S W}$, is estimated by

$$
\dot{m}_{S W, i}=\left\{\left(4.72 \times 10^{-5}\right) f_{S W}\left(T_{B}-36.5\right)\right\}_{i} \quad i=1,2, \ldots, N
$$

$f_{S W, i}=e^{\left(\left(T_{S K, i}-33.7\right) / 10.7\right)}$

In the foregoing equations, a control signal, such as $\left(33.7-T_{S K}\right)$ is set equal to zero, if negative.

\section{HEAT BALANCE}

By performing heat balances on the segmental core, skin, and the clothing layers, the change in these temperatures with time can be predicted. The heat balance equations for segments $\mathrm{i}=1,2, \ldots, \mathrm{N}$ are:

$$
\begin{aligned}
Q_{M T, i}-\left(Q_{R S, i}+Q_{C S, i}\right) & =Q_{S T, C R, i} \\
& =(m \cdot C)_{C R, i} \frac{d T_{C R, i}}{d t} \\
Q_{C S, i}-\left(Q_{E P, i}+Q_{C L, i}\right)= & Q_{S T, S K, i} \\
& =(m \cdot C)_{S K, i} \frac{d T_{S K, i}}{d t} \\
Q_{S C, i}-\left(Q_{C V, i}+Q_{R D, i}\right) & =Q_{S T, C L, i} \\
& =(m . C)_{C L, i} \frac{d T_{C L, i}}{d t}
\end{aligned}
$$

In these equations $Q_{\mathrm{ST}}$ represents the total heat gained or lost.

For small time intervals, $\Delta t$, the rate of change of core temperature, $\mathrm{T}_{\mathrm{CR}}$, skin temperature, $\mathrm{T}_{\mathrm{SK}}$, and clothing temperature, $T_{C L}$, will be approximately constant, so that

$$
\begin{aligned}
& T_{C R, i}(t)=T_{C R, i}(t-\Delta t)+\frac{Q_{S T, C R, i} \cdot \Delta t}{(m C)_{C R, i}} \\
& T_{S K, i}(t)=T_{S K, i}(t-\Delta t)+\frac{Q_{S T, S K, i} \cdot \Delta t}{(m C)_{S K, i}} \\
& T_{C L, i}(t)=T_{C L, i}(t-\Delta t)+\frac{Q_{S T, C L, i} \cdot \Delta t}{(m C)_{C L, i}}
\end{aligned}
$$

Thus, if initial values of $T_{C R}, T_{S K}$, and $T_{C L}$ are given, subsequent values can be calculated from the heat rate terms and heat capacities.

\section{HEATED/VENTILATED SEAT THERMAL MODEL}

The schematic of the all-purpose driver seat is shown in Fig. 3. The seat is designed to augment the standard engine-coolant-based heating system in winter and to provide ventilated cold air to the contact surfaces during the summer to achieve a higher level of comfort. Heat is produced by passing electric current through a heating pad made of carbon fibers placed beneath the leather covering. Ambient air is drawn in using a built-in fan and then flows through spacer material, a heating mat, and the porous leather cover before coming in contact with the driver's body. 


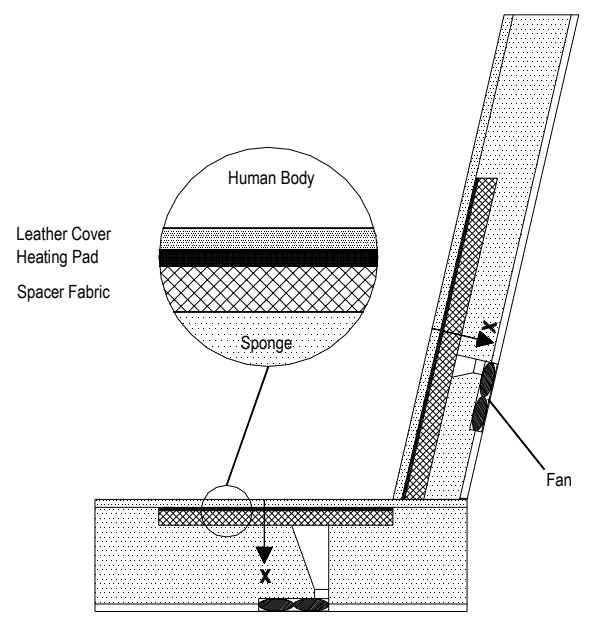

Fig. 3: Schematic of the heated/ventilated seat

Although heat transfer in the seat is a three-dimensional phenomenon, simple transient one-dimensional thermal models are used to predict the time variation of temperature in the seat during the heating and ventilation processes. The assumption of 1-D heat transfer can be justified by considering relatively large thermal resistances in other directions compared to the primary flow direction (x-direction).

The governing equation for the heated seat is

$\frac{\partial^{2} T_{i}}{\partial x_{i}^{2}}+\frac{\dot{Q}_{i}}{k_{i}}=\frac{1}{\alpha_{i}} \frac{\partial T_{i}}{\partial t} \quad i=1,2, \ldots$, No. of seat layers

Equation 19 shows the time variation of temperature across the seat materials. $\dot{Q}$ is the heat generation per unit volume of the heating pad and is zero for other seat materials. $\mathrm{k}$ and $\alpha$ are thermal conductivity and thermal diffusivity $\left(k / \rho C_{P}\right)$ of the seat materials.

The governing equation for ventilation is obtained by writing an energy balance over a small control volume as shown in Fig. 4.

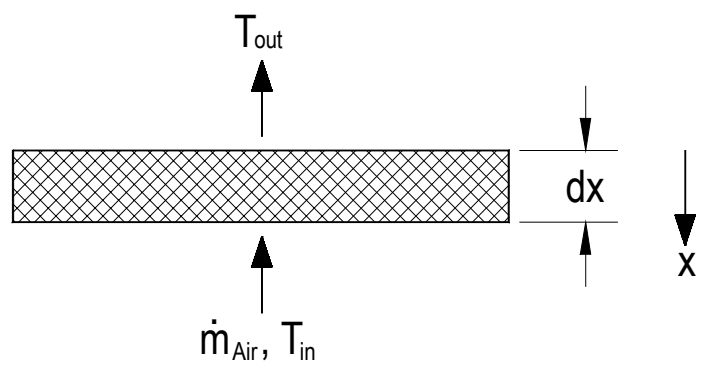

Fig. 4: Energy balance over an element of the seat

Air enters the control volume at $\mathrm{T}_{\mathrm{i}}$, exchanges heat with the seat material and leaves the control volume at $T_{0}$. The change in temperature with time can be estimated using a lumped heat capacity approach as follows
$\frac{T_{o}-T_{i}}{T_{t=0}-T_{i}}=e^{-\beta t}$

where

$$
\beta=\frac{\left(\dot{m} C_{P}\right)_{\text {Air }}}{\left(m C_{P}\right)_{\text {Material }}}
$$

Here, $T_{t=0}$ is the initial seat temperature, $m$ is mass of the seat material in the control volume, $\dot{m}$ is mass flow rate of ventilated air, and $C_{P}$ is the specific heat of the air or the seat material.

Equation 20 is derived based on the following assumptions: (a) heat transfer occurs only in the $x$ direction, (b) the conduction term is negligible compared to convection, (c) the mass of air trapped in the material is negligible in comparison to the mass of the material, and (d) a thermal equilibrium is reached between the air and the material when air leaves the control volume.

Winter simulations are obtained by applying initial and boundary conditions. Equation 19 is discretized implicitly using a finite difference technique. The resulting set of algebraic equations forms a tri-diagonal matrix, which is solved using a Thomas Algorithm. The temperature distributions are updated with time.

Transient temperature profiles due to ventilation are obtained by dividing the seat layers into small control volumes. Calculations are started from the beginning of the spacer material where air is introduced and continued upward to the leather cover. The temperature profiles in the sponge and the leather on the seat back are still governed by conduction and obtained by solving Eq. 19.

\section{THERMAL COMFORT}

The second stage of the thermal comfort modeling is to use the heat transfer data found in the first stage to predict the thermal sensation (comfort) the person would experience. The physiological comfort a person perceives in a car is the result of his or her subjective thermal and moisture sensation. The thermal sensation is created partly due to the driver contact with the seat, and partly due to his/her exposure to the ambient air.

The thermal sensation values can be computed by comparing the rate of heat generation by the body (due to metabolism and a possible shivering) with the rate of heat dissipation that a person would require to maintain thermal neutrality under the specified level of activity, clothing and ambient conditions.

The present model uses Fanger's empirical correlation to estimate the local as well as the overall thermal sensation of a driver. The correlation is 


$$
\begin{array}{r}
T S_{i}=\left\{\left[0.303 \exp \left(\frac{-0.036 Q_{\text {in }}}{A}\right)+0.028\right] \frac{Q_{\text {in }}-Q_{\text {out }}}{A}\right\}_{i} \\
i=1,2, \ldots, N
\end{array}
$$

where $Q_{\text {in }}$ ad $Q_{\text {out }}$ are the rate of heat generation and heat loss from the body segment, respectively. The overall thermal sensation, TS, is calculated by averaging local thermal sensations, $\mathrm{TS}_{\mathrm{i}}$, proportional to the surface areas.

The thermal sensation values obtained by the foregoing equation are interpreted against the thermal sensation scale in Table-2.

\begin{tabular}{cl}
\multicolumn{2}{c}{ Table-2: Scale of thermal sensations } \\
\hline Scale & Thermal Sensation \\
\hline 5 & Painfully hot \\
4 & Very hot \\
3 & Hot \\
2 & Warm \\
1 & Slightly Warm \\
0 & Thermally neutral \\
-1 & Slightly cool \\
-2 & Cool \\
-3 & Cold \\
-4 & Very cold \\
-5 & Painfully cold \\
\hline
\end{tabular}

\section{RESULTS}

In this section transient thermal interactions of a typical driver with the heated/ventilated seat and the ambient air is simulated for severe winter and summer conditions. Throughout the simulations, an average driver of $75 \mathrm{~kg}$, $175 \mathrm{~cm}$ high is considered under moderate traffic conditions. The driver's body is divided into 21 segments, and physical parameters such as mass fraction and skin thickness are assigned for each segment. The driver is wearing a business suit with up to three layers of clothing allowed for every segment. The driver's initial skin and core temperatures are considered to be at 34 and $37^{\circ} \mathrm{C}$, respectively. The initial clothing temperature is taken as a proportional average of the skin and the ambient temperatures. Air movement is considered to be constant at $0.2 \mathrm{~m} / \mathrm{s}$ all around the driver. All simulations are performed over a period of 20 minutes with a time interval of 1 second.

\section{WINTER SIMULATIONS}

Initial cabin temperature and relative humidity are considered to be $-20{ }^{\circ} \mathrm{C}$ and $50 \%$, respectively. It is assumed that the seat initially comes to a thermal equilibrium with the ambient air. Although the cabin air temperature is usually changing exponentially during the warm up period, the air temperature is considered to increase linearly to the comfort level of $22{ }^{\circ} \mathrm{C}$ over a period of 10 minutes for simplicity.
Figures 5a-1,2 show variations of seat cushion and back surface (leather cover) temperatures as a function of time for different heat settings. Variation of the cabin air temperature is also shown in the figures. It takes about 20 minutes for the unheated seat to reach the steady state temperature, which is still well below an acceptable comfortable level. When the heating pad is activated, heat is produced in the resistive carbon fibers at a rate of $1,745,000 \mathrm{~W} / \mathrm{m}^{3}$ and transferred to the leather cover and eventually to the driver's skin. The rate of heat transfer is so fast that the seat surface temperature exceeds $30^{\circ} \mathrm{C}$ in less than 5 minutes. The electrical heater is shut down once its temperature rises above a predefined value (eg. 37,45 , and $55^{\circ} \mathrm{C}$ for the heating, low, medium and high, respectively) and reactivated if its temperature drops more than $2{ }^{\circ} \mathrm{C}$.

Figures $5 b-1,2$ show variations of skin temperatures in contact with the seat cushion and the seat back, respectively. As shown in the figures, the skin temperatures decrease continuously with the unheated seat, however; the rate of skin temperature drop would decrease with time due to shivering stimulated in the corresponding body segments. When the heating system is activated, the skin temperatures drop for the first few minutes due to the seat, clothing and skin's heat capacities. However, the seat heat dissipation can increase skin temperature above $34{ }^{\circ} \mathrm{C}$ very quickly, depending on the seat settings.

Figures $5 c-1,2$ show local heat loss/gain for the body segments in contact with the cushion and back, respectively. As indicated, both segments loose heat quickly at the beginning for all settings however, the rate of heat loss decreases with time due to the body thermoregulatory system and/or the heat transfer from the seat. With the heated seat activated, heat is eventually transferred from the seat to the skin in both regions.

Figures $5 d-1,2$ show local thermal sensations as a function of time. It is clearly shown in these figures that when the seat is unheated the driver will feel very cold at the contact areas for the first few minutes. Even the thermo-regulatory system is unable to bring thermal comfort in a reasonable time period. When the heating system is activated, local thermal sensations increase quickly and the driver will feel warm after about 3 minutes. If the heating system is not shut down after this period the driver will feel localized heating at the contact areas with the seat. At this time the body thermoregulatory system will lower the high thermal sensations to reasonable values through vascular constriction and by producing more evaporation of sweat. The fluctuations in the thermal sensation values are due to the seat built-in on/off controller as described before.

\section{SUMMER SIMULATIONS}

Initial cabin and seat temperatures are considered to be $50{ }^{\circ} \mathrm{C}$. The relative humidity is $50 \%$, and it is assumed that the cabin air conditioning system reduces the air temperature linearly to $22{ }^{\circ} \mathrm{C}$ in 10 minutes. The built-in fans are able to take up to 40 CFM of ambient air and deliver it to the seat cushion and back. 
Figures 6a-1,2 show variations of seat surface temperatures at different ventilation settings as a function of time. Variations of the cabin air temperature with time are also shown in the figures. As indicated, the surface temperature of a non-ventilated seat decreases very slowly and stays well above local skin temperatures for more than 20 minutes of simulations. As a result, the heat is continuously transferred from the seat to the skin and the local skin temperatures rise above the comfort level. With the ventilation system on, local seat surface temperatures drop almost at the same rate as the air temperature and eventually stay a couple of degrees above the steady-state ambient temperature.

Ventilation of warm air through the seat during the first few minutes will increase local skin temperatures however, as soon as the car's A/C reduces the air temperature the seat cools down quickly and ensures a higher level of comfort at the contacted areas. This is clearly shown in Figs. $6 b-1,2$ where the local skin temperatures drop more than $2{ }^{\circ} \mathrm{C}$.

Figures $6 c-1,2$ indicate rates of heat transfer at the contacted areas as a function of time. As seen from the figures, when the body comes in contact with the seat, the rate of heat transfer is very high due to the large temperature gradients at the contact areas. The rate of heat transfer will decrease with time due to the lower temperature gradient and body thermo-regulatory activities. The initial heat flux on the seat is affected by the heat capacity mainly, of the covering material, e.g. leather in this case study. Ventilation of the cabin air through the seat causes the rate of local heat transfer to linearly decrease and its direction to be reversed in 6 minutes of operation. The heat transfer rate approaches a steady-state value soon after the ambient temperature becomes constant. The steady-state heat flux on the seat is affected on one hand by the seat's thermal insulation, mainly created by the inner components of the seat cushion and the backrest and on the other hand by the rate of ventilation. The present study shows little difference between low and medium ventilations rates and almost no difference between the medium and high rates.

Local thermal sensation values at different ventilation settings are shown in Figs. 6d1,2. As seen from the figures, ventilation of air will reduce thermal sensations quickly to the negative territories and a thermally uncomfortable situation will be reached if ventilation continues. Again numerical results show no difference between the medium and high rates of ventilations.

OVERALL THERMAL SENSATION

The body transient overall thermal sensations were calculated using Eq. 21. Local thermal sensations are combined and an average value obtained based on body segmental areas.

Figure 7a shows overall thermal sensations as a function of time for winter simulation. It is clear from the figure that a heated seat will bring thermal comfort to the driver in a shorter period of time, typically between 1 to 2 minutes depending on the setting on the seat. This figure also shows that it is more comfortable to deactivate the heating system after the thermal comfort level is approached.

Figure $7 \mathrm{~b}$ displays transient overall thermal sensations for summer conditions. As seen from the figure, thermal comfort will be achieved at a shorter time with a ventilated seat.

\section{CONCLUSIONS}

A transient physical model is developed to simulate the thermal interactions between an automobile passenger, the cabin environment, and a heated/ventilated seat. The model takes into account the effect of heating and ventilation through the seat on the local and overall thermal sensations. Simulation results indicate that a heated seat will bring thermal comfort to the passenger very quickly in the contact areas which in turn enhance overall thermal sensations. On the other hand, ventilation will bring the seat temperature to that of the ambient air and increase thermal comfort of the passenger.

\section{ACKNOWLEDGMENTS}

The authors would like to acknowledge the financial support of Materials and Manufacturing Ontario (MMO) and the Natural Sciences and Engineering Research Council of Canada (NSERC)

\section{REFERENCES}

ASHRAE. Fundamentals Handbook, 1993. p. 8.6

S. D. Burch, S. Ramadhyani and J.T., Pearson, "Analysis of Passenger Thermal Comfort in an Automobile Under Severe Winter Conditions," ASHRAE Transactions. 1992. 247-257.

T. J. Dohetry, and E. Arens, "Evaluation of the Physiological Bases of Thermal Comfort Models," ASHRAE Transactions, 1988, Vol. 94, Part I, 1371-1385.

P. O. Fanger, "Thermal Comfort: Analysis and Applications in Environmental Engineering," McGraw Hill Co., 1972.

R. A. Haslam, and K.C. Parsons, "An Evaluation of Computer-based Models That Predict Human Responses to the Thermal Environment," ASHRAE Transactions, 1988. Vol. 94, Part 1, 1342-1360.

W. A. Lotens, "Comparison of Thermal Predictive Models for Clothed Humans," ASHRAE Transactions, 1988, Vol. 94, Part 1, 1321-1341. 


\section{CONTACT}

Gholamreza Karimi, Microelectronics Heat Transfer Laboratory, Department of Mechanical Engineering, University of Waterloo, Waterloo, ON, N2L 3G1, Canada

Phone: (519) 888-4567, Ext. 5612

E-Mail: karimir@mhtlab.uwaterloo.ca

\section{NOMENCLATURE}

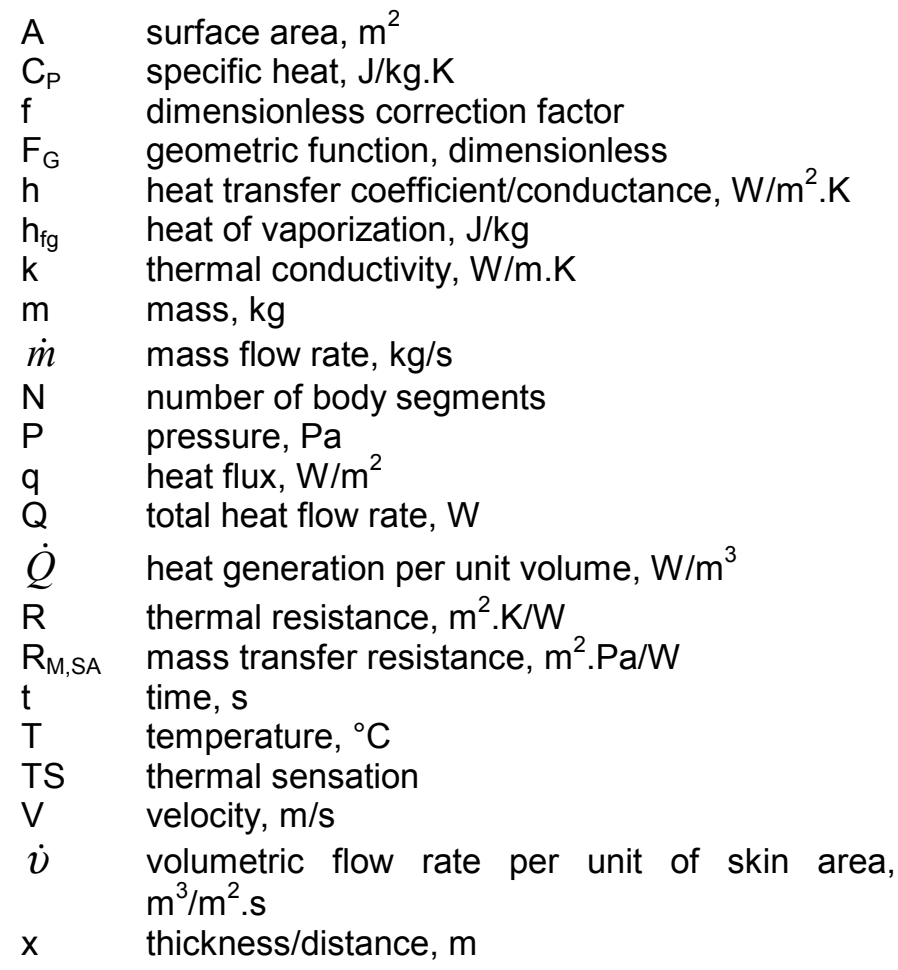

\section{Greek Symbols}

$\alpha \quad$ thermal diffusivity, $\mathrm{m}^{2} / \mathrm{s}$

$\varepsilon \quad$ emissivity

$\omega \quad$ wetness

$\rho \quad$ density, $\mathrm{kg} / \mathrm{m}^{3}$

$\sigma \quad$ Stefan-Boltzmann constant

$\Delta \quad$ difference

\section{Subscripts}

$\begin{array}{ll}\text { A } & \text { ambient air } \\ \text { B } & \text { body } \\ \text { BL } & \text { blood } \\ \text { CA,C-A cloth-to-air } \\ \text { CL } & \text { cloth } \\ \text { CN } & \text { conduction } \\ \text { CR } & \text { core } \\ \text { CS,C-S core-to-skin } \\ \text { CV } & \text { convection } \\ \text { EP } & \text { evaporation } \\ \text { Eff } & \text { effective } \\ \text { i } & \text { inlet/body segment number } \\ \text { MT } & \text { metabolism } \\ \text { O } & \text { outlet } \\ \text { RD } & \text { radiation }\end{array}$

RS respiration

SC,S-C skin-to-cloth

$\mathrm{SH} \quad$ shivering

SK skin

ST storage

SW sweating 

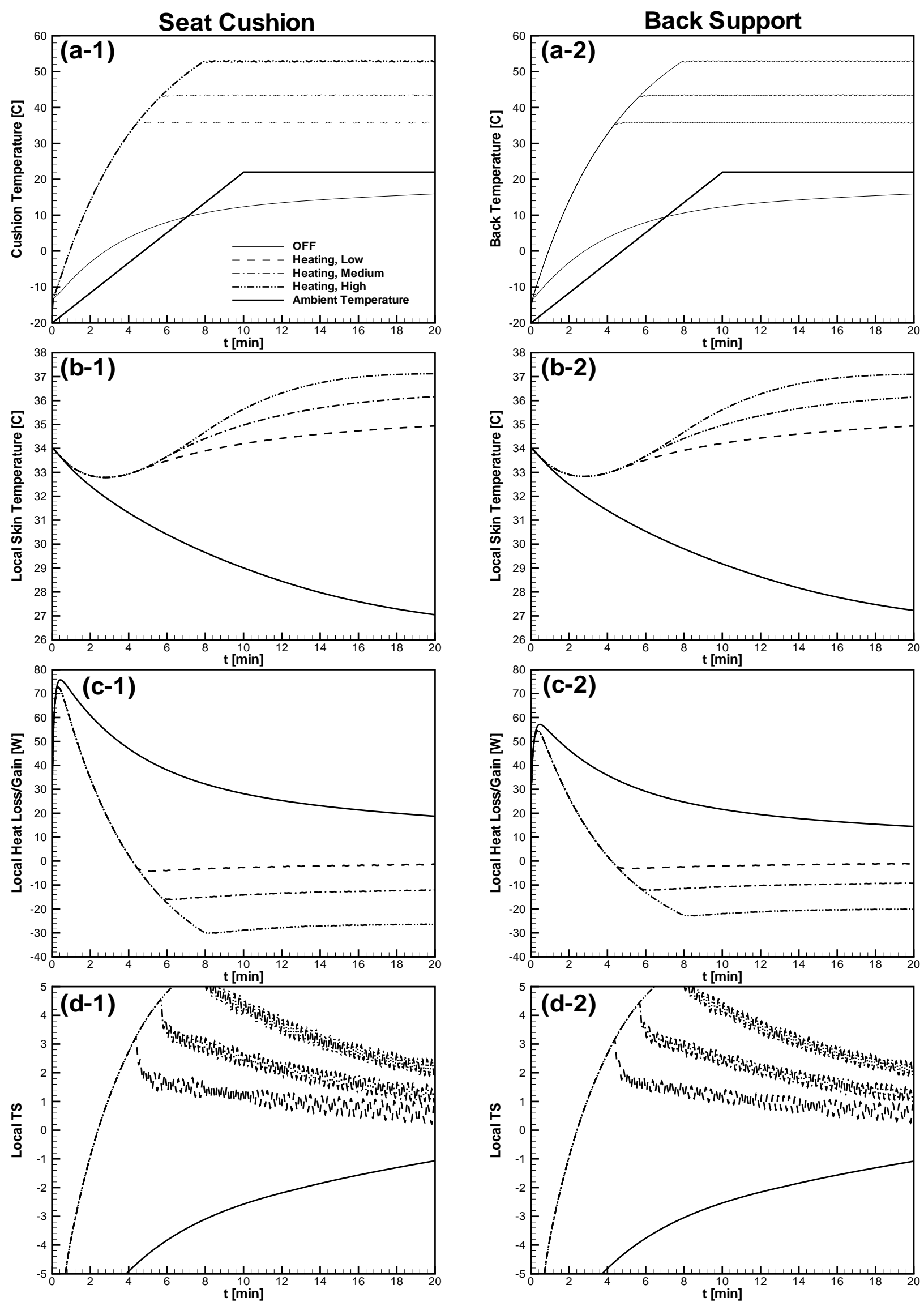

Fig. 5: Time variation of seat temperature, local skin temperatures, local heat losses/gains, and local thermal sensations (TS) as a function of heat settings during winter conditions. (Cushion: LHS figures, Backrest: RHS figures) 

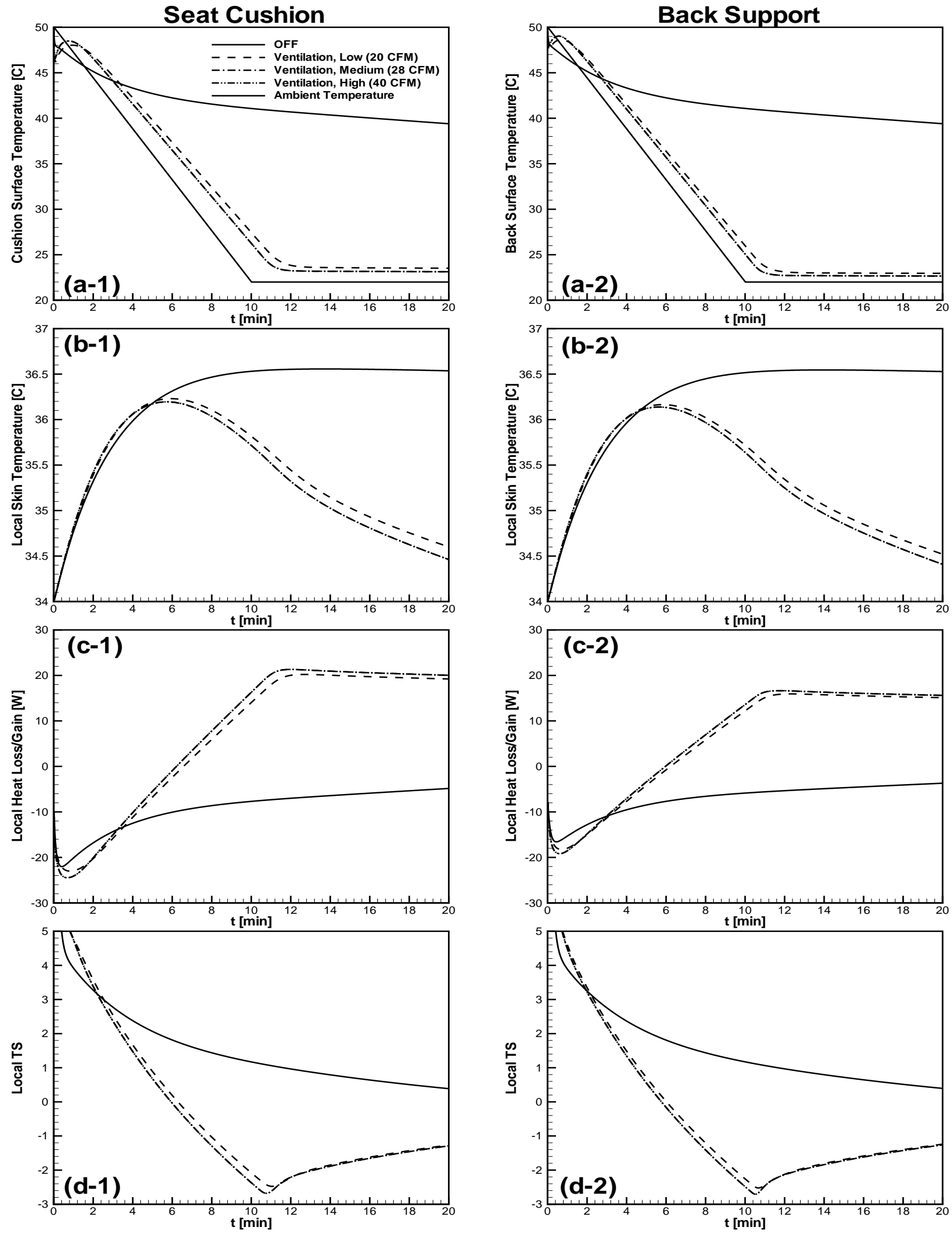

Fig. 6: Time variation of seat temperature, local skin temperatures, local heat losses/gains, and local thermal sensations (TS) as a function of heat settings during summer conditions. (Cushion: LHS figures, Backrest: RHS figures) 

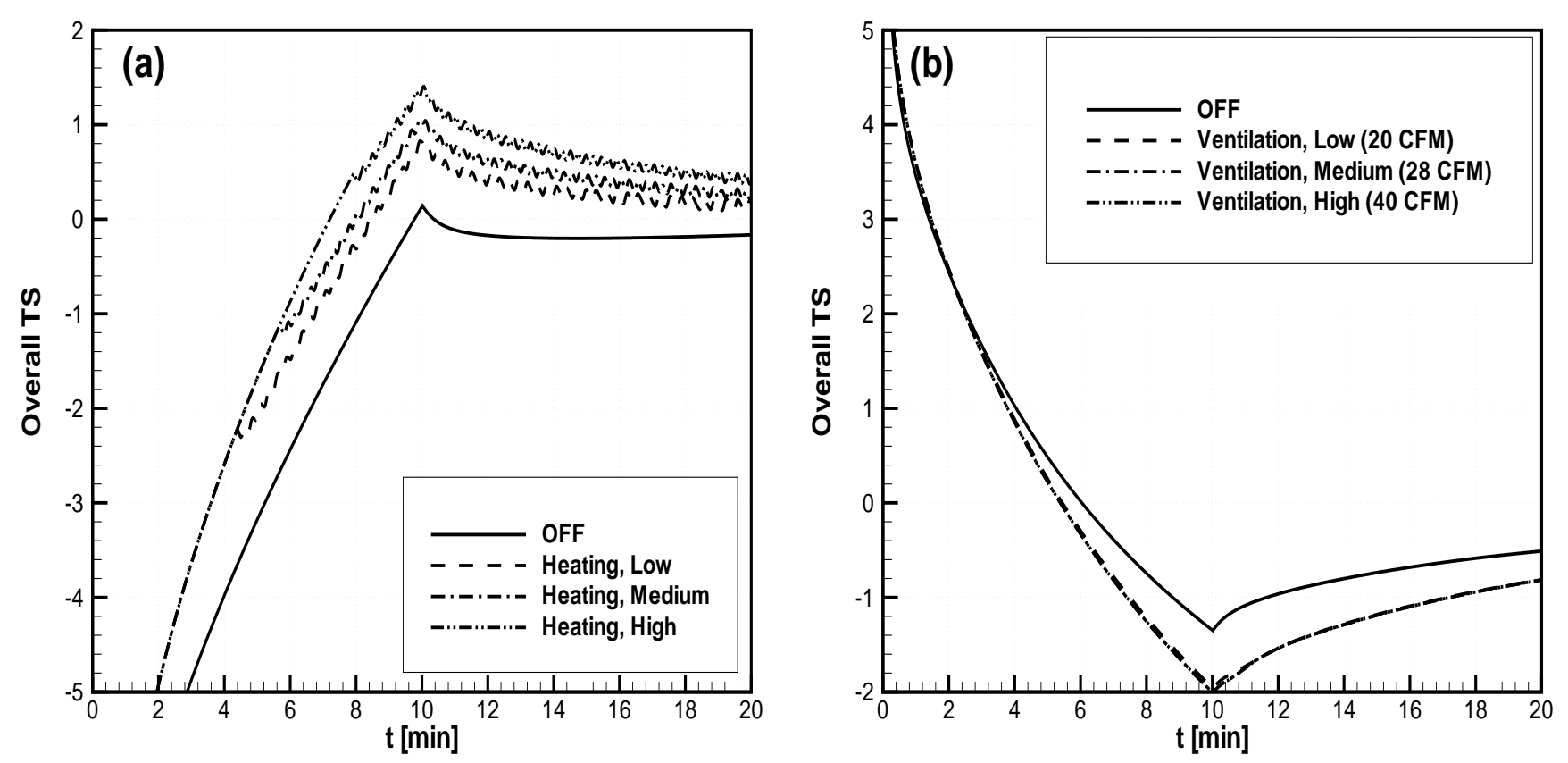

Fig. 7: Driver overall thermal sensations as a function of time. (a) Winter simulation, and (b) Summer simulation 